Ann. Biol. anim. Bioch. Biophys., I973, 13 (3), 363-380.

\title{
ÉVOLUTION CHEZ LA VACHE LAITIÈRE DES TENEURS DE DIFFÉRENTS CONSTITUANTS DU SANG \\ A LA FIN DE LA GESTATION ET AU DÉBUT DE LA LACTATION. RELATIONS AVEC LA SÉCRÉTION DES MATIÈRES GRASSES DU LAIT
}

\author{
B. RÉMOND, R. TOULLEC* et M. JOURNET \\ avec la collaboration technique de Michelle Chassagne, \\ Renée Lefaivre, B. Marquis et Louise Toullec \\ Station de Recherches sur l'Élevage des Ruminants, \\ Centre de Recherches de Clermont-Ferrand, I. N.R. A., \\ Saint Genès Champanelle, 63110 Beaumont \\ *Laboratoire de Recherches de la Chaire de Zootechnie, \\ Centre de Recherches de Rennes, \\ 65, Rue de Saint-Brieuc \\ 35042 Rennes Cedex
}

\section{RÉSUMÉ}

Chez 3 vaches dont l'état nutritionnel était très différent, nous avons décrit pendant les 3 dernières semaines de gestation et les 6 premières semaines de lactation, l'évolution de la concentration des acides gras des 4 principales classes de lipides plasmatiques et de leur composition en acides gras, ainsi que l'évolution de la concentration des corps cétoniques sanguins, du glucose et des protéines totales plasmatiques. Nous avons essayé de relier cela à la quantité et à la composition des matières grasses sécrétées dans le lait, ainsi qu'à l'état nutritionnel des animaux.

La concentration des acides gras des triglycérides, des phospholipides et des esters du cholestérol, des acides gras totaux, ainsi que celle des protéines et du glucose a diminué à la fin de la gestation, été minimale au début de la lactation et augmenté ensuite ; celle des acides gras libres et des corps cétoniques a varié en sens inverse.

La part des acides gras libres dans les acides gras totaux du plasma, normalement d'environ 5 p. I00, a atteint 20 p. IOO après le vêlage chez la vache la plus sous-alimentée. Pendant le même temps, la proportion des acides gras des triglycérides était 8 fois plus faible.

Des différents constituants sanguins que nous avons mesurés, c'est le glucose et les acides gras libres qui ont été le mieux liés (intra- et inter-vaches) au bilan énergétique.

La quantité de matières grasses sécrétée dans le lait et le taux buryteux ont été positivement liés à la concentration des acides gras libres plasmatiques.

La composition en acides gras des acides gras libres a été semblable à celle des triglycérides, mais différente de celle des phospholipides et des esters du cholestérol. Dans les acides gras libres, les triglycérides et les phospholipides, $\mathrm{C}_{18: 0}$ a varié dans le même sens que le bilan énergétique et $\mathrm{C}_{18: 1}$ a varié en sens inverse. 
Dans le lait, la proportion des acides gras longs (à i 8 atomes de carbone) a été d'autant plus élevée au début de la lactation que le déficit énergétique était plus important (ou que la concentration des acides gras libres était plus grande) ; elle a diminué avec l'avancement de la lactation.

Les résultats ont été comparés à ceux d'autres auteurs, et discutés à propos de la synthèse des matières grasses en particulier.

\section{INTRODUCTION}

Au début de la lactation, la teneur du lait en matières grasses et la proportion relative de ses différents acides gras évoluent rapidement (DECAEN et ADDA, 1970) ; l'importance et la durée de ces évolutions semblent liées, du moins chez la Vache, à celles de l'état nutritionnel des animaux et de la teneur de leur plasma en acides gras libres (DECAEN et JOURNET, I967; RÉMOND et JOURNETr, I970). Mais on connaît encore très mal la participation des différentes classes de lipides sanguins à la synthèse des matières grasses du lait au début de la lactation. L'évolution de leur teneur plasmatique a été peu étudiée et de façon peu détaillée (cf. études de LENNON et MIXNER, I957; Duncan et GarTon, I963; HarTManN et LASCELliES, I964; Varman et SchuLTZ, I968) ; de plus, parmi ces auteurs, seuls les derniers ont aussi mesuré la composition en acides gras des différentes classes de lipides.

Nous avons donc voulu étudier de façon plus précise l'évolution de la concentration des différentes classes de lipides plasmatiques de 3 vaches à la fin de la gestation et au début de la lactation, et celle de leur composition en acides gras, en faisant varier l'état nutritionnel des animaux dont on sait déjà qu'il détermine en grande partie la teneur du plasma en acides gras libres.

\section{MATÉRIEL ET MÉTHODES}

\section{Animaux et alimentation}

Les 3 vaches utilisées, désignées par les lettres $\mathrm{A}, \mathrm{B}$ et $\mathrm{C}$ dans la suite de l'exposé, étaient de race Française Frisonne Pie Noive et allaient effectuer leur $2^{\mathrm{e}}(\mathrm{C})$ ou leur $3^{\mathrm{e}}$ lactation (A et B). De 4 semaines avant le vêlage à 6 semaines après, elles ont reçu chaque jour une ration de base constituée de foin de ray-grass $(4 \mathrm{~kg})$, de luzerne déshydratée compactée $(3 \mathrm{~kg})$ et de pulpes sèches de betteraves $(2 \mathrm{~kg})$; la vache A a reçu $4 \mathrm{~kg}$ de luzerne compactée et $3 \mathrm{~kg}$ de pulpes à partir du vêlage. L'aliment concentré a été distribué en quantité telle que les apports d'énergie excédaient les besoins avant et après le vêlage chez la vache $\mathrm{A}$, qu'ils étaient légèrement insuffisants pendant les mêmes périodes chez la vache $B$, et qu'ils étaient très insuffisants chez la vache $C$. Les animaux étaients traits 2 fois par jour et les quantités ingérées étaient mesurées 6 jours par semaine.

Le bilan énergétique a été calculé à partir des quantités d'aliments ingérées et de leur valeur énergétique pour les apports, et des recommandations de LEROY (I949) pour les besoins. La valeur énergétique du foin a été déduite de la digestibilité de sa matière organique mesurée avec des moutons, par la formule de BREIREM (I954); celle de la luzerne déshydratée a été estimée d'après sa composition chimique par les tables de Demarguiliy (I970); celle des pulpes et de l'aliment concentré a été tirée des tables de la valeur alimentaire des aliments de LEROY.

\section{Échantillonnage du lait et du sang, et analyses}

Un échantillon de beurre a été constitué pour chaque animal avec le lait des 2 traites de la journée, les $4^{\mathrm{e}}, 8$ ou $9^{\mathrm{e}}, 15^{\mathrm{e}}, 22$ ou $23^{\mathrm{e}}$, et 36 ou $37^{\mathrm{e}}$ jours de la lactation. La composition en acides gras (AG) des échantillons ainsi obtenus a été mesurée par chromatographie en phase gazeuse des esters butyliques (GHADAKl, I968). 
Des échantillons de sang (environ too $\mathrm{ml}$ ) ont été prélevés dans des flacons contenant de 1'héparine, une fois par semaine au cours des 3 semaines qui précédaient le vêlage, et les mêmes jours que les échantillons de beurre étaient constitués après le vêlage. Ils étaient obtenus dans la veine jugulaire, le matin juste avant la distribution des aliments. Le plasma était aussitôt séparé par centrifugation et placé à - $15^{\circ} \mathrm{C}$ dans des flacons de polypropylène jusqu'à l'analyse.

Sur le plasma, nous avons mesuré la concentration du glucose par la méthode à la glucoseoxydase (MICHEL, I97I), celle des protéines totales par la méthode du Biuret adaptée à l'autoanalyseur Technicon, celle des corps cétoniques totaux par la méthode de Procos (I96I), celle des acides gras libres par la méthode de Dole et MeINERTz (I960), celle des lipides totaux (LT) et des acides gras (AG) des 4 principales fractions lipidiques (acides gras libres (AGL) ; triglycérides (TG) ; esters du cholestérol (EC) ; phospholipides (PL) et la composition en AG de ces 4 classes de lipides.

La concentration des LT a été mesurée en triple, avec ro $\mathrm{ml}$ de plasma par répétition, par la méthode de Folch, Lees et Sloane Staniey (I957). La concentration dans le plasma des $\mathrm{AG}$ des différentes fractions lipidiques et leur composition ont été mesurées simultanément grâce à l'utilisation d'étalons internes contenant de l'acide heptadécanoïque.

Nous avons procédé de la manière suivante : les 3 extraits lipidiques obtenus par la méthode de Folch, Lees et Sloane-Stanley (I957) ont été dissous dans du chloroforme, rassemblés dans une fiole jaugée et le volume a été ajusté à $100 \mathrm{ml}$. La solution des lipides était ensuite divisée en 2 parties égales $a$ et $b$. La solution $a$ était utilisée telle quelle dans la suite de l'analyse. A la solution $b$, on ajoutait des volumes déterminés de solutions contenant de's quantités connues d'heptadécanoate de cholestérol, de triheptadécanoate de glycérol, d'acide heptadécanoïque et de monoheptadécanoate de glycérol (MoRRIs, 1967) ; ces étalons internes migraient respectivement en même temps que les EC, les TG, les AGL et les PL en chromatographie sur couche mince.

L'heptadécanoate de cholestérol a été synthétisé à partir d'heptadécanoate de méthyle et d'acétate de cholestérol, selon la méthode de Mahadevan et Lundberg (I962). Le monoheptadécanoate de glycérol a été obtenu en chauffant à $230^{\circ} \mathrm{C}$, pendant $\mathrm{I} h 20$, sous barbotage d'azote, ro $\mathrm{g}$ de glycérol et Io $\mathrm{g}$ d'acide heptadécanoïque (FLANZY, I969) ; il a ensuite été purifié sur colonne d'acide silicique, le triheptadécanoate et le diheptadécanoate étant d'abord élués ensemble par un mélange d'éther de pétrole et d'éther éthylique $(75 / 25 ; \mathrm{V} / \mathrm{V})$ puis le monoheptadécanoate par de l'éther éthylique pur.

Les solutions $a$ et $b$ étaient ensuite concentrées par évaporation sous vide et les 4 classes de lipides qu'elles contenaient étaient séparées par chromatographie sur couche mince $(200 \mu$ d'épaisseur) de gel de silice PF 254-366 (Merck), en réalisant 2 migrations successives à l'aide d'un mélange d'éther de pétrole, d'éther éthylique et d'acide formique $(8 \mathrm{o} / \mathrm{I} / \mathrm{I})$. Les 4 classes de lipides étaient repérées à la lumière ultraviolette et les 4 bandes d'acide silicique qui les contenaient étaient grattées et recueillies dans 4 ballons pour la méthylation; cette dernière était effectué au bain-marie à $80^{\circ} \mathrm{C}$, sous rétrigérant à reflux, à l'aide d'un mélange de méthanol, de chloroforme et d'acide sulfurique (I00/100/2 pour les AGL et roo/Ioo/5 pour les EC, les PL et les TG). La durée de la méthylation était d'une heure pour les AGL et les TG, de 2 h pour les EC et de $2 \mathrm{~h}$ 30 pour les PL; en effet, nous avons constaté qu'il est indispensable d'appliquer des temps de méthylation plus longs pour obtenir le rapport théorique entre l'acide heptadécanoïque et les AG des EC et des PL, lorsque l'on fait méthyler ensemble des quantités connues d'acide heptadécanoïque et de palmitate de cholestérol ou de phospholipides de soja.

Les esters méthyliques étaient extraits par de l'éther de pétrole, lavés à l'eau distillée jusqu'à neutralisation, amenés à sec, puis dissous dans I $\mathrm{ml}$ de chloroforme et conservés à - I $5^{\circ} \mathrm{C}$ en présence d'hydroquinone jusqu'à l'analyse par chromatographie en phase gazeuse. Cette dernière a été effectuée à l'aide d'un Aerograph $204 \mathrm{~B}$ muni de 2 détecteurs à ionisation de flamme. Les colonnes, de $2,5 \mathrm{~m}$ de long et de $2 \mathrm{~mm}$ de diamètre intérieur, étaient remplies de chromosorb W 80-10o mesh imprégné à $16 \mathrm{p}$. Ioo de succinate de diéthylène glycol. La température de l'injecteur et du détecteur était de $220^{\circ} \mathrm{C}$ et celle du four de colonne de I $75^{\circ} \mathrm{C}$. Les débits d'hydrogène et d'azote étaient respectivement de 20 et $25 \mathrm{ml} / \mathrm{mn}$. La surface des pics a été mesurée à l'aide d'un intégrateur électronique Disc. Les teneurs en acide heptadécanoïque des $A G$ des classes de lipides (I à 4 p. IOO) mesurées dans l'échantillon $a$, permettaient de corriger les valeurs correspondantes de l'échantillon $b$, avant de calculer le poids des AG de chaque classe de lipides.

Pour éviter la présence d'artefacts, il était nécessaire de prendre de nombreuses précautions concernant notamment les solvants (distillation) et la verrerie (lavage au mélange sulfochromique en évitant l'utilisation de tout détergent, méthylation préalable à blanc dans les ballons utilisés pour cette opération...). Les artefacts a pportés par l'échantillon de gel de silice que nous avons utilisé pour la chromatographie sur couche mince ne semblaient pas dépasser I $2 \mu \mathrm{g}$ par bande récupérée et n'avaient donc pas d'influence notable sur les mesures de quantité ou de composition d'AG, les quantités minima déposées sur les plaques étant de l'ordre de $600 \mu \mathrm{g}$ 
(TG et AGL) ; cependant, cet apport peut ne pas être négligeable avec certains lots de gels de silice, d'après Carreau, Lapous et Raulin (rg69).

Les résultats ont été traités par analyse de covariance (SNEDECoR et CochraN, I957); à partir des résultats intermédiaires de cette analyse, nous avons calculé les coefficients de corrélation intra-vaches et inter-vaches.

\section{RÉSULTATS}

Le bilan énergétique, calculé en soustrayant les besoins des apports, a toujours été positif pour la vache $A$; il a été négatif jusqu'à la troisième semaine de lactation pour les 2 autres vaches, mais de façon plus importante pour la C que pour la B (fig. 2). La quantité de lait à $4 \mathrm{p}$. Ioo de matières grasses produite par jour a été en moyenne au cours des 5 premières semaines de lactation de I I,I, 22,8 et $25,5 \mathrm{~kg}$ pour les vaches $\mathrm{A}, \mathrm{B}$ et $\mathrm{C}$ respectivement.

Le taux butyreux des 3 premières semaines de lactation et la diminution du taux butyreux de la $\mathbf{I}^{\mathrm{re}}$ à la $5^{\mathrm{e}}$ semaine ont été d'autant plus élevés que le bilan énergétique était déficitaire. La production de matières grasses a été maximale au cours de la $2^{\text {e }}$ semaine de lactation pour les 3 vaches.

\section{Évolution avant et après le vêlage de la concentration dans le plasma} des lipides et des acides gras totaux, du glucose et des protéines (fig. I)

La concentration des $\mathrm{L}^{\mathrm{T}} \mathrm{T}$ mesurée par la méthode de FoLCH, LEES et SLOANESTANLEY (I957) a peu varié avec le vêlage et a augmenté après. La somme des concentrations des AG, mesurées par chromatographie, des 4 classes de lipides (AGL, TG, PL et $\mathrm{EC}$ ) a été minimale au début de la lactation et a également augmenté ensuite. La diminution au moment du vêlage porte sur 3 fractions (TG, PL et EC); elle est cependant plus importante pour les TG dont la concentration a été de 2 à 4 fois moins élevée (selon les vaches) aux environs du $4^{\mathrm{e}}$ jour de la lactation que pendant la $2^{\mathrm{e}}$ ou la $3^{\text {e }}$ semaine qui précédait le vêlage. L'accroissement de la concentration des AG après le vêlage a été plus important pour les $\mathrm{EC}$ que pour les $\mathrm{PL}$, et plus important pour les PL que pour les TG. Six semaines après le vêlage, la concentration des AG des TG était encore très inférieure à celle observée avant le vêlage, alors que celle des $\mathrm{EC}$ et des $\mathrm{PL}$ était très supérieure.

La concentration des AGL a augmenté à la fin de la gestation mais surtout au début de la lactation pour les vaches qui étaient fortement sous-alimentées ( $\mathrm{B}$ et $\mathrm{C}$ ) ; elle a été maximale entre la $\mathrm{I}^{\mathrm{r}} \mathrm{e}$ et la $3^{\mathbf{e}}$ semaine de lactation, et a donc évolué en sens inverse de la concentration des $\mathrm{AG}$ des 3 autres fractions lipidiques. L'amplitude de l'évolution a été d'autant plus importante que le bilan énergétique était plus négatif au début de la lactation.

Les AG des AGL + TG ont constitué plus de Io p. Ioo des acides gras totaux (AGT) en général et 23 p. roo au maximum (tabl. I). Ceux des PL et des EC ont constitué le reste, pour moitié chacun environ.

Les teneurs du sang en corps cétoniques totaux (acétone + acéto-acétale $+\beta$-hydroxybutyrate) et en acétone + acétoacétate ont également augmenté après 

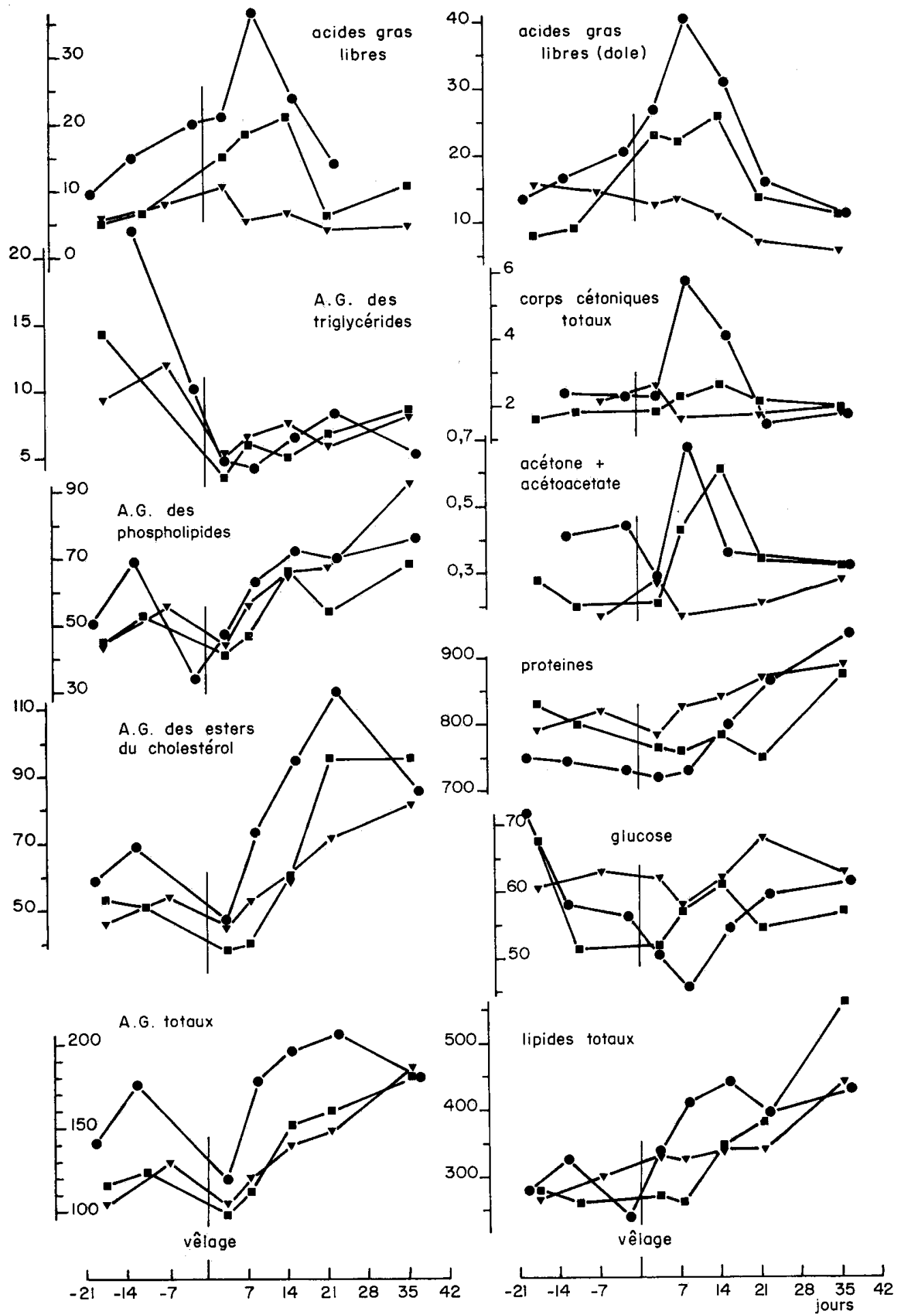

FI. I. - Evolution en fin de gestation et en début de lactation de la concentration de différents constituants du sang ou du plasma (milligramme par $100 \mathrm{ml}$ )

V Vache A Vache B Vache C 
le vêlage pour être maximales entre la $I^{\mathrm{r}}$ et la $3^{\mathbf{e}}$ semaine de lactation pour les vaches les plus sous-alimentées. Cinq à six semaines après le vêlage, elles sont revenues à des valeurs semblables à celles qui étaient observées avant le vêlage.

\section{TABLEAU I}

Contribution des acides gras de chaque classe de lipides aux acides gras totaux du plasma (p. 10o)

\begin{tabular}{|c|c|c|c|c|c|c|c|c|c|c|}
\hline \multirow[t]{2}{*}{ Vache } & \multicolumn{2}{|c|}{ Source des acides gras } & \multicolumn{8}{|c|}{ Pourcentage de chaque classe de lipides } \\
\hline & & Jour $\left({ }^{1}\right) \rightarrow$ & -18 & & -7 & 4 & 8 & 15 & 22 & 36 \\
\hline \multirow{4}{*}{ A } & Acides gras libres & $\ldots \ldots$ & 5,2 & & 6,1 & 9,9 & 4,7 & 4,6 & 2,5 & 2,3 \\
\hline & Triglycérides ... & & 8,8 & & 9,1 & 4,8 & 5,4 & 5,2 & 3,9 & 4,1 \\
\hline & Phospholipides .. & $\ldots$ & 42,3 & & 43,1 & 42,4 & 46,2 & 46,6 & 45,4 & 49,7 \\
\hline & Esters de cholesté & rol ... & 43,7 & & 41,6 & 42,7 & 43,5 & 43,3 & 48,0 & 43,9 \\
\hline \multirow{5}{*}{$\mathrm{B}$} & & Jour $(1) \rightarrow$ & -18 & $-11^{*}$ & & 4 & 8 & 15 & 22 & 36 \\
\hline & Acides gras libres & $\ldots$ & 4,1 & 5,3 & & 15,3 & 16,5 & 13,8 & 3,6 & 5,6 \\
\hline & Triglycérides $\ldots$ & & 12,1 & 11,4 & & 3,4 & 5,3 & 3,3 & 4,1 & 4,6 \\
\hline & Phospholipides .. & $\ldots \ldots \ldots$ & 38,3 & 42,1 & & 41,9 & 42,2 & 43,2 & 33,3 & 37,3 \\
\hline & Esters du cholesté & rol $\ldots .$. & 45,4 & 41,1 & & 39,1 & 36,0 & 39,5 & 58,8 & 52,2 \\
\hline \multirow{5}{*}{$\mathrm{C}$} & & Jour $\left({ }^{1}\right) \rightarrow$ & $-20^{* *}$ & -13 & & 4 & 9 & 16 & 23 & $37 * * *$ \\
\hline & Acides gras libres & $\cdots$ & 6,8 & 8,6 & & 17,4 & 20,4 & 11,9 & 6,6 & 7,7 \\
\hline & Triglycérides ... & & 15,6 & 12,6 & & 4,0 & 2,4 & 3,3 & 4,0 & 2,9 \\
\hline & Phospholipides .. & $\ldots \ldots$ & 35,9 & 39,4 & & 39,4 & 35,7 & 36,4 & 33,8 & 42,1 \\
\hline & Esters du cholesté & Érol ... & 41,8 & 39,3 & & 39,0 & 41,2 & 48,1 & 55,4 & 47,3 \\
\hline
\end{tabular}

La contribution des acides gras des 4 classes de lipides a été calculée en admettant que : la concentration des triglycérides était la même que celle de la semaine précédente $\left(^{*}\right)$ ou suivante $\left(^{* *}\right)$; la concentration des acides gras libres était la même que celle de la semaine précédente $\left.{ }^{* * *}\right)$.

(1) Jour avant (一) ou après le vêlage.

Les concentrations des protéines totales plasmatiques et du glucose sanguin ont évolué dans le même sens que celle des LT et en sens inverse de celle des AGL, comme le montrent la figure I et les corrélations intra-vaches entre ces concentrations (tab1. 2) En particulier, les concentrations du glucose et des protéines ont été minimales I à 2 semaines après le vêlage lorsque la concentration des AGL était maximale.

Avec le bilan énergétique, la teneur du plasma en AGL a été liée étroitement et négativement aussi bien intra-vaches (en fonction du stade physiologique) qu'intervaches, alors que les teneurs en $\mathrm{AG}$ des TG et des $\mathrm{EC}$, en glucose et en protéines ont été liées plus ou moins étroitement mais positivement (intra-vaches ou inter-vaches).

\section{Composition en acides gras des différentes classes de lipides plasmatiques (tabl. 3)}

Les AG des AGL, et des TG contenaient une proportion semblable et élevée de $\mathrm{C}_{16: 0}, \mathrm{C}_{18: 0}$ et $\mathrm{C}_{18: 1}$. Comparativement, les $\mathrm{AG}$ des $\mathrm{PL}$, se sont caractérisés par une proportion plus faible de $C_{18: 1}$, et surtout par une proportion beaucoup plus élevée ( 4 à 


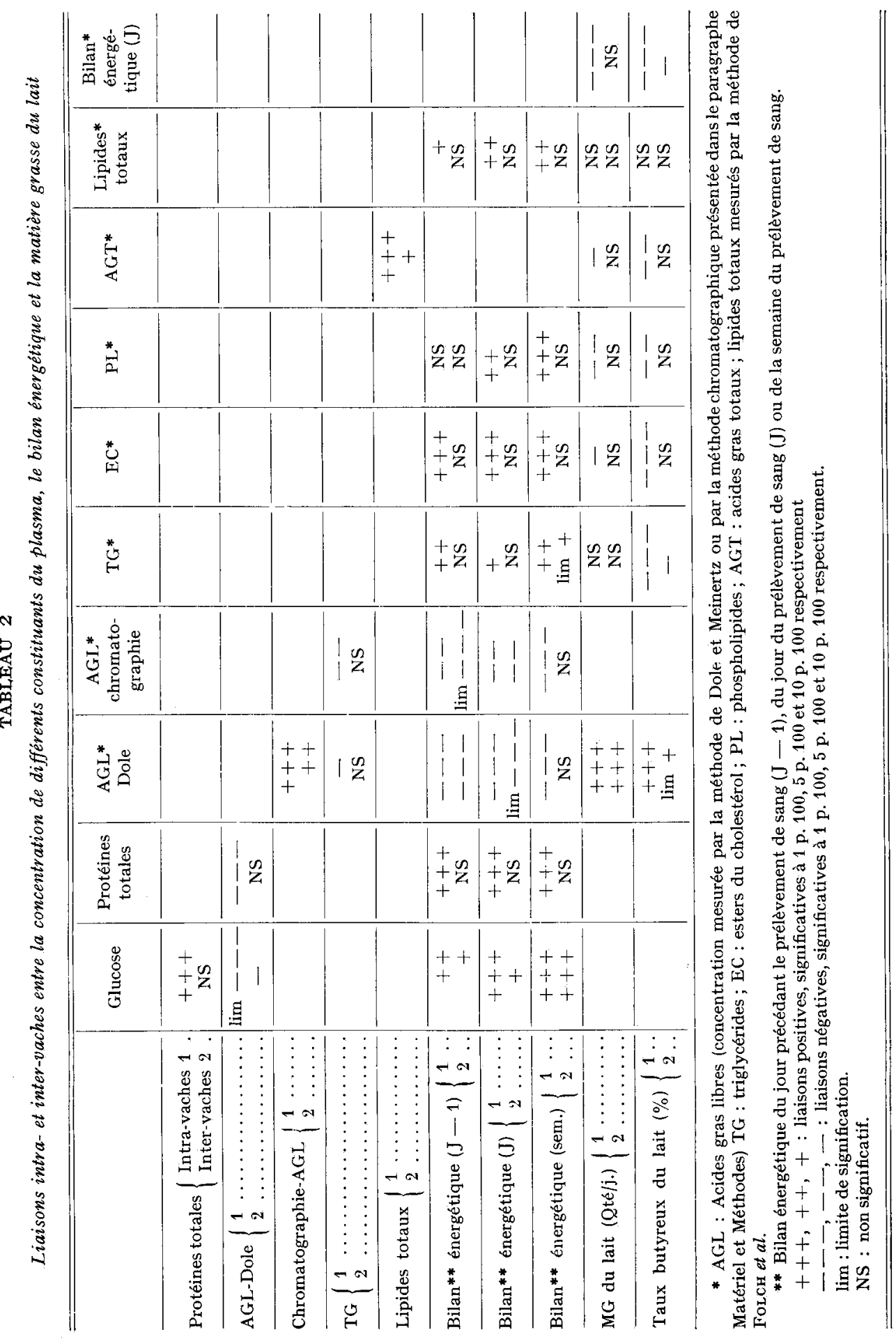


Composition en acides gras des (en poids p. Ioo de

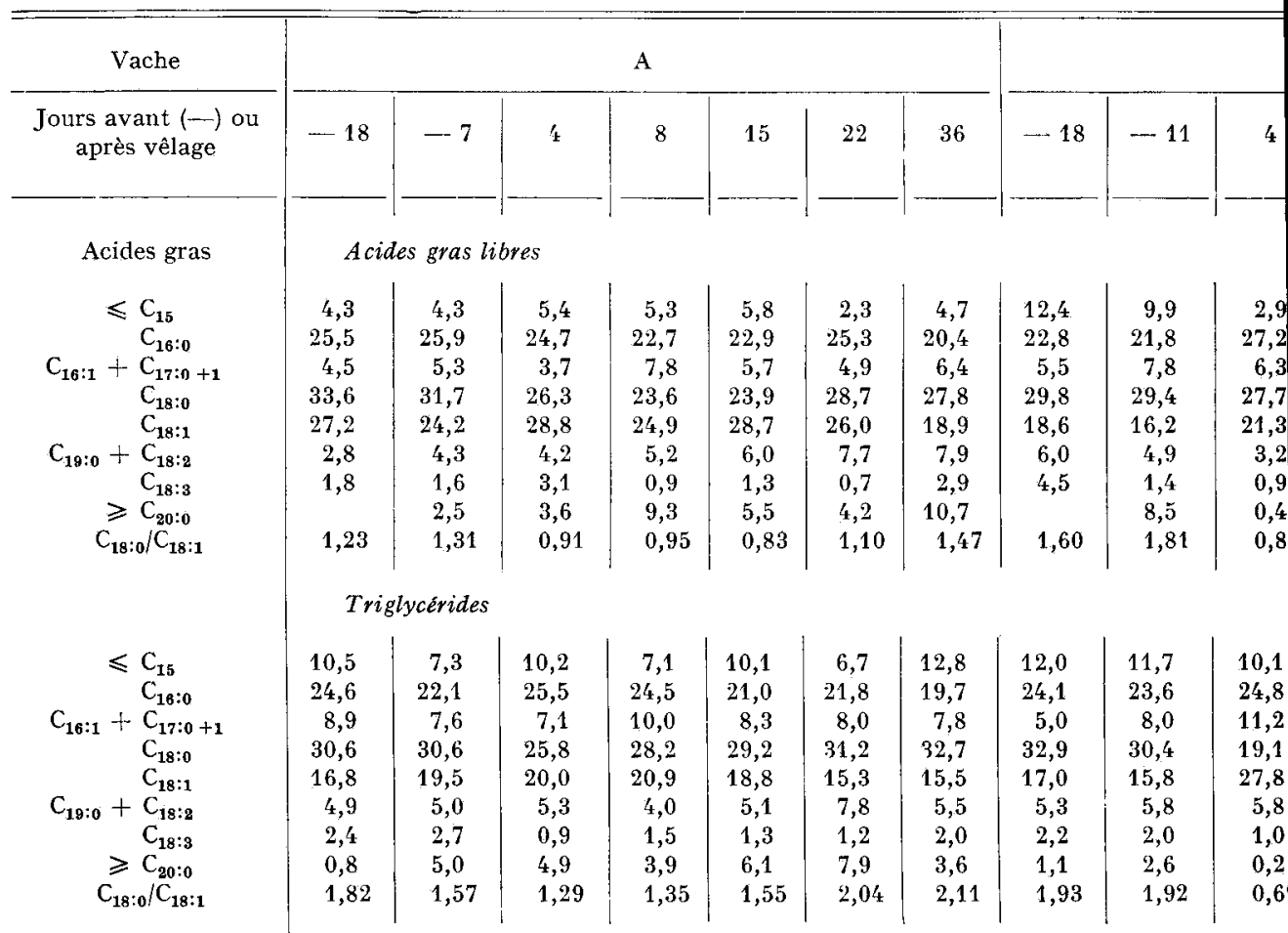

Phospholipides

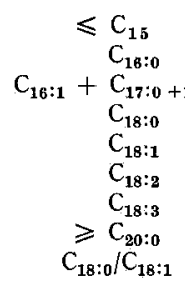

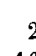

\begin{tabular}{r|r}
2,8 & 2,4 \\
16,3 & 19,7 \\
4,2 & 4,0 \\
23,1 & 21,8 \\
11,7 & 11,8 \\
25,4 & 28,6 \\
6,6 & 4,2 \\
9,7 & 7,5 \\
1,97 & 1,85
\end{tabular}

1,5
22,7
3,8
22,4
14,7
19,9
2,0
13,0
1,52

\begin{tabular}{|c|c|}
3,2 & 2,9 \\
17,5 & 16,4 \\
5,1 & 5,8 \\
20,6 & 22,2 \\
11,5 & 9,4 \\
27,0 & 28,5 \\
3,5 & 2,5 \\
11,7 & 12,3 \\
1,79 & 2,36 \\
&
\end{tabular}

\begin{tabular}{r|}
3,9 \\
14,8 \\
6,9 \\
21,6 \\
9,4 \\
27,0 \\
2,9 \\
13,2 \\
2,30 \\
\hline
\end{tabular}

\begin{tabular}{|c|r|}
4,9 & 2,6 \\
18,0 & 16,5 \\
2,7 & 6,3 \\
23,5 & 23,5 \\
11,2 & 12,1 \\
24,0 & 21,6 \\
6,0 & 5,7 \\
10,0 & 11,7 \\
2,10 & 1,94
\end{tabular}

0,9 22,7 4,2 24,6 19,2 19,0 3,8 5,6 1,2

Esters au cholestérol

$$
\begin{aligned}
& \leqslant C_{15} \\
& C_{16: 0} \\
& C_{16: 1}+ C_{17: 0+1} \\
& C_{18: 0} \\
& C_{18: 1} \\
& C_{18: 2} \\
& C_{18: 3} \\
& \geqslant C_{20: 0} \\
& C_{18: 2} / C_{18: 3}
\end{aligned}
$$

\begin{tabular}{c|c|c}
6,0 & 6,6 & 5,1 \\
6,0 & 5,6 & 5,5 \\
3,6 & 4,2 & 4,4 \\
0,9 & 0,9 & 0,4 \\
4,1 & 4,0 & 5,5 \\
48,0 & 55,8 & $\mathbf{5 2 , 7}$ \\
21,1 & 17,3 & 15,8 \\
10,5 & 5,5 & 10,5 \\
2,27 & 3,22 & 3,33
\end{tabular}

\begin{tabular}{c|c|}
$\mathbf{4 , 0}$ & 3,5 \\
5,2 & 5,0 \\
4,3 & 3,5 \\
0,5 & 0,2 \\
6,2 & 4,7 \\
58,0 & 63,3 \\
16,1 & 14,2 \\
5,5 & 5,5 \\
3,60 & 4,46
\end{tabular}

\begin{tabular}{c|c|}
3,6 & 4,7 \\
4,5 & 4,5 \\
2,9 & 3,1 \\
0,4 & 0,4 \\
3,5 & 4,1 \\
63,0 & 62,9 \\
13,3 & 13,5 \\
8,9 & 6,8 \\
4,73 & 4,66
\end{tabular}

\begin{tabular}{r|r}
8,3 & 8,6 \\
7,0 & 5,8 \\
4,0 & 4,2 \\
1,0 & 1,2 \\
5,8 & 5,5 \\
44,2 & 43,2 \\
23,3 & 21,2 \\
6,3 & 10,3 \\
1,90 & 2,04
\end{tabular}

6,5 7,5 4,8 


\begin{tabular}{|c|c|c|c|c|c|c|c|c|c|c|c|}
\hline \multirow[b]{2}{*}{8} & \multirow[b]{2}{*}{15} & \multirow[b]{2}{*}{22} & \multirow[b]{2}{*}{36} & \multicolumn{7}{|c|}{$\mathrm{C}$} & \multirow[b]{2}{*}{37} \\
\hline & & & & -20 & -13 & -2 & 4 & 9 & 16 & 23 & \\
\hline 8,8 & 5,9 & 6,0 & $\mathbf{5 , 2}$ & 3,6 & 7,1 & 5,4 & 6,7 & 4,2 & 3,0 & 5,1 & 7,9 \\
\hline, 2 & 22,5 & 22,8 & 22,6 & 22,7 & 24,3 & 21,6 & 28,0 & 21,1 & 24,8 & 21,8 & 24,2 \\
\hline 7,1 & 5,8 & 6,8 & 6,4 & 6,1 & 7,0 & 9,9 & 6,3 & 7,5 & 6,0 & 6,9 & 4,5 \\
\hline, \pm 6 & 28,0 & 28,1 & 33,8 & 28,7 & 25,4 & 20,7 & 21,2 & 18,7 & 20,6 & 16,9 & 25,5 \\
\hline 2,9 & 24,8 & 23,9 & 19,9 & 19,8 & 24,8 & 34,0 & 26,5 & 35,2 & 41,0 & 40,3 & 24,9 \\
\hline$\beta, 1$ & 3,2 & 5,5 & 5,1 & 2,4 & 2,9 & 3,8 & 2,4 & 6,4 & 2,9 & 3,4 & 8,5 \\
\hline 0,9 & 0,7 & 0,4 & 1,3 & 1,4 & 1,5 & 2,6 & 1,1 & 3,7 & 0,8 & 3,0 & 2,0 \\
\hline 3,0 & 8,6 & 6,8 & 5,7 & & 7,0 & 1,8 & 7,7 & 3,0 & 0,8 & 2,6 & 2,1 \\
\hline 0,75 & 1,13 & 1,17 & 1,70 & 1,45 & 1,02 & 0,61 & 0,80 & 0,58 & 0,50 & 0,42 & 1,02 \\
\hline 0,4 & 9,9 & 7,8 & 7,7 & 9,0 & 16,4 & 9,8 & 11,8 & 7,9 & $1 l_{t}, 1$ & 19,7 & 7,4 \\
\hline 0,0 & 21,2 & 23,8 & 21,3 & 24,8 & 22,8 & 24,2 & 27,6 & 25,7 & 26,6 & 20,0 & 25,7 \\
\hline 7,2 & 8,1 & 7,7 & 5,1 & 9,9 & 12,8 & 10,8 & 11,4 & 11,6 & 10,0 & 11,4 & 4,7 \\
\hline 1,1 & 24,2 & 31,3 & 41,8 & 30,7 & 21,8 & 21,2 & 13,3 & 13,7 & 11,8 & 10,7 & 28,5 \\
\hline 1,6 & 19,2 & 18,5 & 17,9 & 15,2 & 13,4 & 22,4 & 26,9 & 27,4 & 28,4 & 25,0 & 24,7 \\
\hline 7,7 & 4,6 & 5,6 & 3,4 & 3,9 & 2,8 & 7,7 & 5,6 & 8,4 & 5,3 & 4,3 & 6,1 \\
\hline 1,5 & 3,8 & 1,1 & 1,0 & 2,5 & 2,6 & 3,0 & 1,0 & 0,8 & 2,2 & 1,6 & 1,4 \\
\hline 0,7 & 8,8 & 4,3 & 1,5 & 4,0 & 7,8 & 0,6 & 2,0 & 4,4 & 1,7 & 7,8 & 1,2 \\
\hline 0,67 & 1,26 & 1,69 & 2,33 & 2,02 & 1,63 & 0,95 & 0,49 & 0,50 & 0,41 & 0,43 & 1,15 \\
\hline 3,8 & 4,1 & 2,4 & 2,2 & 3,8 & 2,2 & 2,8 & 1,7 & 1,0 & 2,1 & 2,6 & 3,9 \\
\hline 1,0 & 20,5 & 18,4 & 19,8 & 20,6 & 22,1 & 25,9 & 25,4 & 24,4 & 24,2 & 21,2 & 19,5 \\
\hline 3,2 & 4,4 & 5,5 & 4,5 & 4,6 & 4,2 & 4,2 & 4,2 & 3,9 & 4,9 & 3,8 & 5,6 \\
\hline 3,8 & 23,7 & 21,3 & 23,6 & 24,5 & 25,7 & 24,8 & 22,1 & 24,7 & 18,4 & 22,0 & 23,1 \\
\hline 0,1 & 16,5 & 14,2 & 12,9 & 11,8 & 13,1 & 16,2 & 21,3 & 22,9 & 22,7 & 26,1 & 15,8 \\
\hline 6,7 & 21,5 & 24,3 & 26,7 & 16,0 & 20,7 & 16,5 & 16,7 & 12,0 & 19,3 & 20,1 & 25,7 \\
\hline 3,3 & 2,4 & 2,3 & 1,5 & 4,6 & 3,6 & 3,3 & 2,5 & 2,1 & 2,0 & 2,2 & 1,9 \\
\hline 7,8 & 6,6 & 11,5 & 8,4 & 14,5 & 8,0 & 6,0 & 6,0 & 7,7 & 6,0 & 1,8 & 4,0 \\
\hline 1,18 & 1,44 & $1 ; 50$ & 1,83 & 2,08 & 1,96 & 1,53 & 1,04 & 1,08 & 0,81 & 0,84 & 1,46 \\
\hline 4,2 & 3,5 & 3,2 & 4,4 & 7,2 & 6,3 & & 4,4 & 14,2 & 4,3 & 7,8 & 5,8 \\
\hline 7,7 & 6,0 & 4,2 & 4,0 & 6,6 & 7,0 & & 7,5 & 6,7 & 9,0 & 8,2 & 5,2 \\
\hline 4,0 & 3,3 & 3,5 & 3,9 & 4,2 & 5,0 & & 5,2 & 5,4 & 6,2 & 8,2 & 5,2 \\
\hline 0,7 & 0,5 & 0,4 & 0,5 & 1,2 & 1,3 & & 1,0 & 0,8 & 1,4 & 0,8 & 0,6 \\
\hline 7,4 & 6,6 & 5,6 & 4,6 & 7,9 & 7,7 & & 10,0 & 9,1 & 16,5 & 13,1 & 8,1 \\
\hline 4,0 & 53,4 & 57,3 & 66,2 & 40,7 & 48,4 & & 40,8 & 37,2 & 45,4 & 48,2 & 58,8 \\
\hline 7,3 & 16,2 & 10,2 & 9,7 & 19,2 & 16,5 & & 18,1 & 16,4 & 10,7 & 9,9 & 10,9 \\
\hline 4,4 & 10,2 & 15,3 & 6,6 & 12,8 & 7,8 & & 12,8 & 10,5 & 6,6 & 3,5 & 5,0 \\
\hline 2,54 & 3,30 & 5,62 & 6,82 & 2,12 & 2,93 & & 2,25 & 2,27 & 4,24 & 4,87 & 5,39 \\
\hline
\end{tabular}


5 fois plus) de $\mathrm{C}_{18: 2}$. Les $\mathrm{AG}$ des $\mathrm{E} C$ se sont caractérisés par une très forte proportion de $\mathrm{C}_{18: 2}$ et $\mathrm{C}_{18: 3}$.

La proportion de $\mathrm{C}_{\text {18:0 }}$ dans les différentes classes de lipides a peu varié avec l'avancement de la lactation, sauf peut-être dans les AGL où elle a été parfois plus importante juste après le vêlage. Les variations les plus importantes ont porté sur les teneurs en $\mathrm{C}_{18: 0}$ et $\mathrm{C}_{\text {18:1 }}$ dans les AGL, les TG et également les PL. La proportion de $\mathrm{C}_{18}$ : a évolué avec le stade de lactation en sens inverse du bilan énergétique : elle a été faible avant le vêlage maximum après, chez les vaches en bilan énergétique négatif ( $\mathrm{B}$ et $\mathrm{C}$ ), et elle a diminué jusqu'à la $5^{-6^{e}}$ semaine de lactation, au fur et à mesure que le bilan énergétique augmentait. La proportion de $\mathrm{C}_{18: 0}$ a varié plus faiblement (surtout dans les PL) mais en sens inverse de la proportion de $\mathrm{C}_{18: 1}$, de sorte que le rapport $\mathrm{C}_{18: 0} / \mathrm{C}_{18: 1}$ a varié dans des proportions considérables. Pour la vache la plus sous-alimentée en début de lactation, il a varié dans la proportion de I à 2 dans les $A G L$ et les $P L$ et de I à 3 dans les TG. D'une vache à l'autre la proportion de $\mathrm{C}_{\mathbf{1} \text { : } 1}$ et le rapport $\mathrm{C}_{18: 0} / \mathrm{C}_{1 \text { ::1 }}$ dans les $\mathrm{AGL}$, les $\mathrm{TG}$ et les $\mathrm{PL}$ ont également varié en fonction du bilan énergétique, de la même façon qu'intra-vaches. Les variations les plus importantes de la composition des $\mathrm{AG}$ des $\mathrm{EC}$ ont porté sur le $\mathrm{C}_{18: 2}$ qui a augmenté entre la $\mathrm{I}^{\mathrm{re}}$ et la 6 e semaine de lactation et sur le $\mathrm{C}_{18: 3}$ qui a varié en sens inverse. Ces variations ont été observées pour les 3 vaches indépendamment semble-t-il du bilan énergétique.

La proportion des $\mathrm{AG}$ à $I 8$ atomes de carbones $\left(\mathrm{C}_{18: 0}+\mathrm{C}_{18: 1}+\mathrm{C}_{18: 2}+\mathrm{C}_{18: 3}\right)$ dans les AGT a été voisine de 60 p. Ioo (un peu moins dans les AGL et les TG, et un peu plus dans les PL). Elle a peu varié en fonction du stade physiologique et entre animaux, et en fonction du bilan énergétique ; elle a légèrement augmenté en passant de 55 à $60 \mathrm{p}$. Ioo dans les AGL lorsque le bilan énergétique diminuait.

\section{Sécrétion des matières grasses du lait (tabl. 4)}

La composition en AG des matières grasses du lait a évolué avec le stade de lactation mais l'amplitude de variation a été très différente selon les vaches. En moyenne, la proportion des $\mathrm{AG}$ à courte chaîne ( 4 à $\mathrm{I} 2$ atomes de carbone par molécule) a augmenté avec l'avancement de la lactation et celle des $\mathrm{AG}$ à chaîne longue (I8 atomes de carbone) a diminué. La proportion des $\mathrm{AG}$ à chaîne courte a été d'autant plus élevée et celle des $A G$ à chaîne longue a été d'autant plus faible que le bilan énergétique était élevé, aussi bien intra-vaches qu'inter-vaches. Ces résultats sont en accord avec ceux obtenus par DECAEN et ADDA (I970) et DECAEN et JouRNET (I967).

Le rapport $\mathrm{C}_{18: 0} / \mathrm{C}_{18: 1}$ a été beaucoup plus faible dans le lait que dans le plasma : 2 à 3 fois plus environ que dans les AGL, 2 à 4 fois plus que dans les TG, et 3 à 5 fois plus que dans les PL. Inter-vaches, il a varié dans le même sens dans les matières grasses du lait et dans les AGL, les TG et les PL plasmatiques : il a été d'autant plus faible que le bilan énergétique était fortement négatif. Avec le stade de lactation il n'a pas varié de façon bien définie.

Le taux butyreux a diminué après le vêlage au fur et à mesure que le bilan énergétique augmentait (fig. 2 ), et cette variation a été due aux acides gras longs ( $r$ intravaches $=0,83^{2} ; \mathrm{P}<0,0 \mathrm{o}$ ) ; elle s'est faite dans le même sens que la teneur en AGL, plasmatiques mais en sens inverse de celle en $\mathrm{AG}$ des $\mathrm{TG}$, comme l'indiquent les liaisons portées dans le tableau 2. Inter-vaches, le taux butyreux a été également 


\begin{tabular}{|c|c|c|c|c|}
\hline \multirow{16}{*}{ 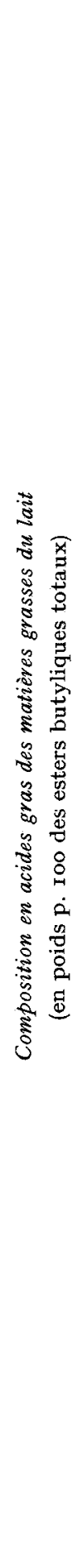 } & \multirow{5}{*}{ u } & $\infty$ & 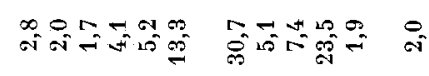 & 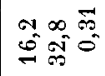 \\
\hline & & ล̊ & ô of & 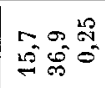 \\
\hline & & $\stackrel{2}{\sim}$ & 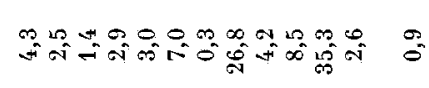 & 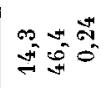 \\
\hline & & $\theta$ & 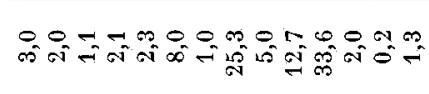 & 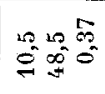 \\
\hline & & 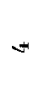 & 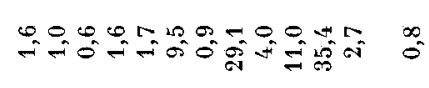 & 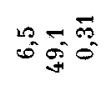 \\
\hline & \multirow{5}{*}{$\infty$} & $\mathscr{~}$ & 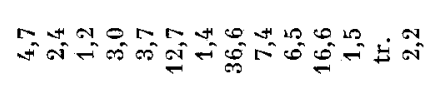 & 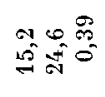 \\
\hline & & $\mathcal{N}$ & 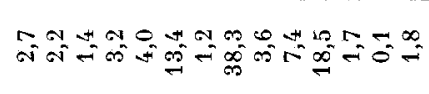 & 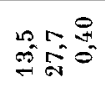 \\
\hline & & $\stackrel{20}{\rightarrow}$ & 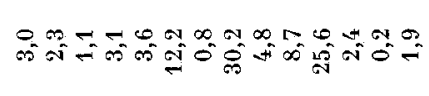 & 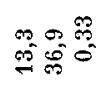 \\
\hline & & $\infty$ & 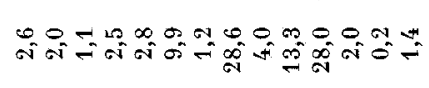 & $=\frac{\pi}{9}=0$ \\
\hline & & 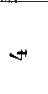 & 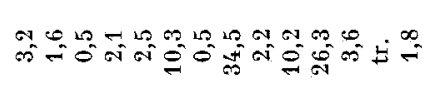 & 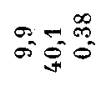 \\
\hline & \multirow{5}{*}{$\varangle$} & $\mathscr{\oplus}$ & 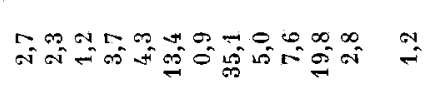 & 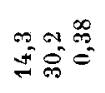 \\
\hline & & $\stackrel{N}{N}$ & 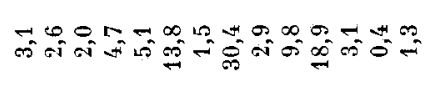 & 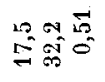 \\
\hline & & 22 & 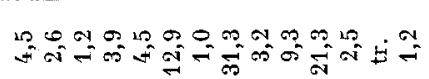 & $\Leftrightarrow=\frac{5}{0}$ \\
\hline & & $\infty$ & 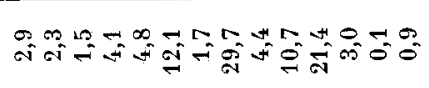 & 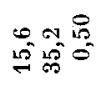 \\
\hline & & $\rightarrow$ & 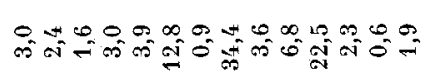 & 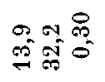 \\
\hline & $\begin{array}{l}\frac{0}{0} \\
\frac{\pi}{0} \\
>\end{array}$ & 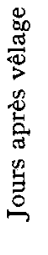 & 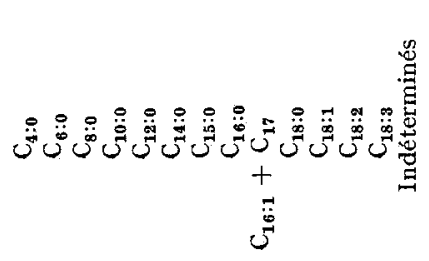 & 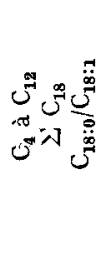 \\
\hline
\end{tabular}


d'autant plus élevé que le bilan énergétique était plus négatif, mais les liaisons établies avec la teneur en acides gras longs du lait $(r=+0,8 \mathrm{II})$ et la teneur en AGL plasmatiques $(r=+0,986)$ ont été positives mais pas significatives. La liaison avec la teneur en $\mathrm{AG}$ des $\mathrm{TG}$ a été négative et significative au niveau Io p. roo (tab1. 2).
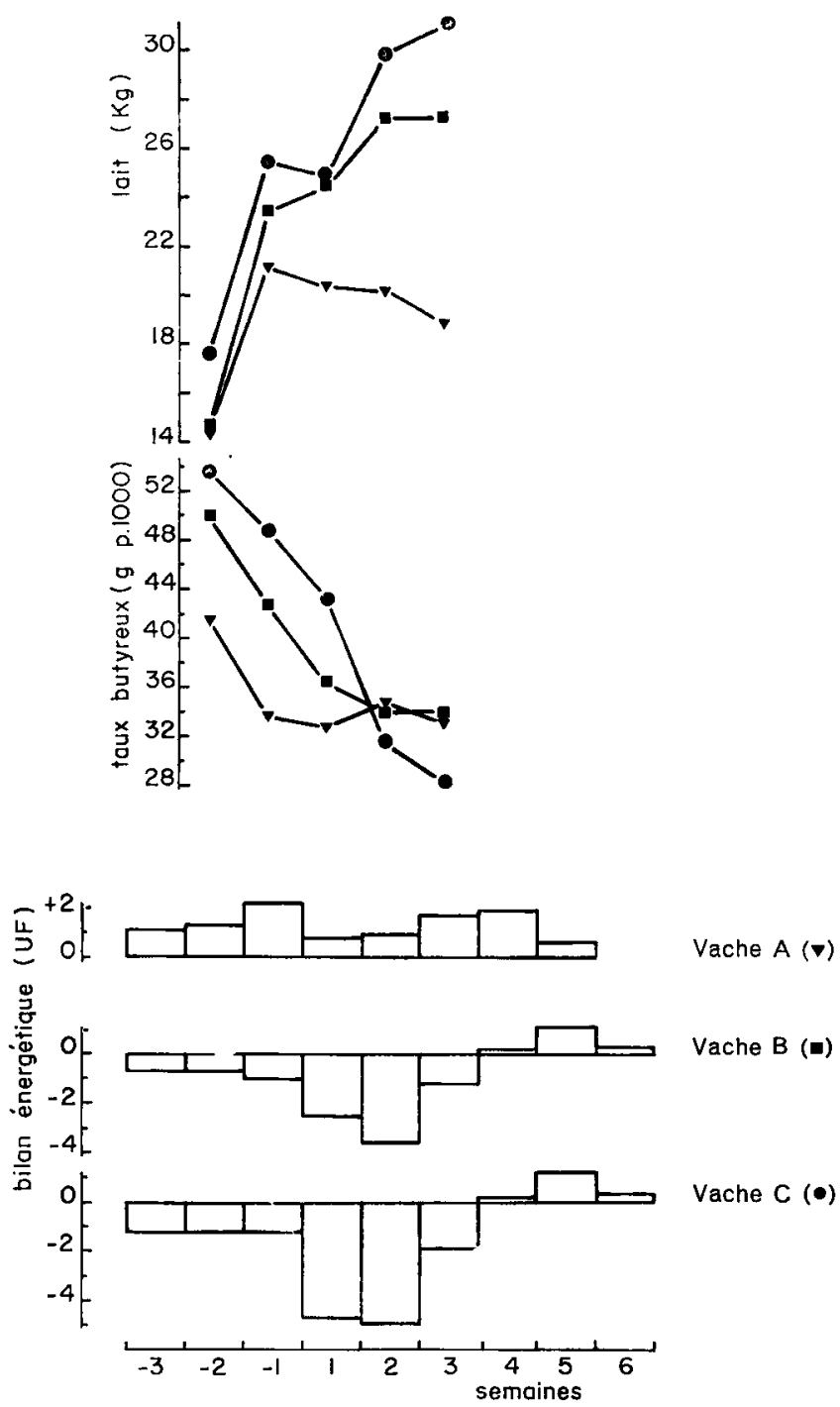

Fic. 2. - Evolution de la quantité de lait produite, du taux butyreux et du bilan énergétique

\section{DISCUSSION}

Les liaisons très étroites (tabl. 2) entre les teneurs du plasma en AGL mesurées par la méthode de DolE et MEINERTz (I960) et par la méthode chromatographique que nous avons exposée, confirment la valeur de cette dernière pour mesurer la concen- 
tration des différentes fractions lipidiques dans le plasma, compte tenu du fait que la séparation des différentes classes lipidiques était bonne; néanmoins les PL étaient confondus avec les monoglycérides.

\section{Les fractions lipidiques du plasma}

Les évolutions à la fin de la gestation et au début de la lactation des concentrations dans le plasma des AGT ou des $\mathrm{L} T$, et des $\mathrm{AG}$ des différentes fractions lipidiques ont été semblables à celles présentées par DunCAN et GARTON (I963) et HARTMANN et LASCELIES (I964) ; mais nous avons observé qu'au début de la lactation les AGL, pouvaient contribuer au total des $A G$ dans une proportion beaucoup plus importante que ne l'avaient observé Duncan et GARTON (20 p. Ioo au lieu de 8 p. Ioo). Cette différence doit être attribuée au fait que nos prélèvements de sang ont été faits plus tôt après le vêlage et sur des vaches probablement plus sous-alimentées. D'ailleurs, d'après les résultats de VARMAN et SchulTz (I 968) obtenus sur des vaches fortes productrices au début de la lactation, on peut calculer que la proportion des AGL dans les AGT du plasma artériel était de l'ordre de I7 p. Ioo.

Nous avons confirmé l'existence de liaisons étroites et négatives entre le bilan énergétique et la concentration des AGL, plasmatiques, mais nous avons égalcment observé une liaison positive entre le bilan énergétique et la teneur en $\mathrm{AG}$ des TG (et aussi des EC). Cette liaison n'implique cependant pas une relation de cause à effet. FISHER et al. (I97I) ont en effet montré qu'une sous-alimentation au cours de la $3^{\mathrm{e}}$ semaine de lactation n'avait pas empêché une augmentation dans le sang de la concentration des esters totaux, des PL et du cholestérol ; et il a fallu un jeûne complet de 4 jours en milieu de lactation pour que HARTMANN et LASCELLES (I965) observent une diminution de la concentration des EC et des PL. On peut ainsi penser que l'évolution importante de la lipémie et de la concentration des 3 principales fractions lipidiques, TG, PL et $\mathrm{EC}$, mais surtout de ces deux dernières, avant et après le vêlage est sous un contrôle hormonal relativement indépendant de l'état nutritionnel, en rapport avec le stade physiologique des animaux; en revanche, la teneur en AGL plasmatiques semble étroitement dépendre directement ou indirectement de l'état nutritionnel.

La composition en AG des différentes fractions lipidiques est assez semblable à celle trouvée par d'autres auteurs (Evans et al., I96r ; Duncan et GARToN, r963 ; LEAT, I966 ; LEAT et HALL, I968) mais sensiblement différente de celle trouvée par DELAGE et FEHR (I 967 ) sur des chèvres.

Mais les faits les plus remarquables sont probablement 1'importance et le parallélisme des variations du pourcentage de $C_{18: 0}$ et de $C_{18: 1}$ dans les $A G I_{1}$, les TG et même les $\mathrm{PL}$ (les EC contiennent très peu de ces deux $A G$ ).

\section{Origine des matières grasses du lait}

Les liaisons étroites observées par DECAEN et JouRNET (I967) au début de la lactation (et confirmées par cette étude) entre le bilan énergétique et la teneur en matières grasses du lait, en acides gras longs surtout, permettaient de penser que ceuxci provenaient de la mobilisation des lipides corporels.

La proportion élevée de $C_{18: 1}$ observée à la fois dans le lait et dans les 3 fractions 
lipidiques du plasma (AGL, TG et $\mathrm{PL}$ ) lorsque le bilan énergétique est négatif confirme encore que l'animal fait bien appel à ses réserves lipidiques qui sont particulièrement riches en $\mathrm{C}_{18: 1}$ (HILdiTcr et WiL,iams, rg64).

Les corrélations intra-vaches élevées observées entre la proportion d'acides gras longs du lait et la teneur en AGL plasmatiques $(r=0,84 \mathrm{I} ; \mathrm{P}<0, \mathrm{or})$ contribuent à faire penser que ces derniers sont les précurseurs directs des acides gras longs du lait chez les vaches laitières normalement sous-alimentées en début de lactation. KRONFELD (I965) avait d'ailleurs observé des différences artérioveineuses d'AGL d'autant plus élevées que la teneur du plasma artériel était élevée : alors que ANNison et al. (I967) n'avaient pas observé de différences artérioveineuses pour de faibles teneurs en AGL plasmatiques chez des chèvres en milieu de lactation. Pendant le jeûne, ANNISON et al. (I968) avaient également observé des différences artérioveineuses.

On peut se demander quel est, en début de lactation, le rôle joué par les TG plasmatiques dont on sait que certains sont normalement les principaux précurseurs des acides gras longs du lait. Nous observons qu'ils ont une teneur minimale juste après le vêlage au moment où la quantité d'acides gras longs sécrétée dans le lait est maximale. On pourrait attribuer cette faible concentration à un prélèvement très important par la mamelle. Mais cette hypothèse est infirmée par les résultats obtenus par VARMan et SchuLTz (I968) et Schwal,M et al. (I972) sur des vaches en début de lactation, et qui présentaient des concentrations en AGL et en TG plasmatiques tout à fait semblables à celles de nos vaches les plus sous-alimentées. Ces auteurs ont en effet observé des différences artérioveineuses très faibles des TG et des différences artérioveineuses d'AGL 3 fois plus élevées que celles des TG.

On peut donc penser que la diminution de la concentration des TG après le vêlage résulte de l'hydrolyse hors de la mamelle des TG des lipoprotéines et des chylomicrons; selon Tove (I965), la lipoprotéine lipase mammaire est libérée dans le plasma efférent de la glande et sa concentration augmente considérablement au moment du vêlage. Il est donc possible que les AGL plasmatiques qui seraient donc bien les principaux précurseurs des matières grasses du lait en début de lactation, proviennent en partie de 1'hydrolyse des TG; cela pourrait expliquer les corrélations négatives observées par Schwalm et al. (I972) entre les différences artérioveineuses d'AGL et de TG, et les corrélations négatives que nous avons obtenues entre la teneur du plasma en TG et le taux butyreux du lait.

Il semble bien, d'après les variations de la composition en AG des TG et des AGL, que les deux principaux acides gras longs prélevés soient le $C_{18: 0}$ et le $C_{18: 1}$. BARRY et al. (r963) avaient montré que les acides gras longs des lipoprotéines à faible densité étaient prélevés dans la proportion de $60 \mathrm{p}$. Ioo d'acides saturés (principalement $\mathrm{C}_{18: 0}$ ) et de $40 \mathrm{p}$. Ioo d'acides insaturés (principalement $\mathrm{C}_{18: 1}$ ). Nous observons en effet, lorsque les vaches sont en bilan énergétique équilibré, que la proportion de $\mathrm{C}_{18: 0}$ est environ 2 fois plus élevée que celle de $\mathrm{C}_{18: 1}$ (dans les AGL, et les TG) ; mais aussi qu'en cas de sous-alimentation, la tendance est inversée ; c'est ce que montre bien la liaison négative observée entre le rapport $\mathrm{C}_{18: 0} / \mathrm{C}_{\mathbf{1 8 : 1}}$ dans les $A G L$ et les TG plasmatiques, et la teneur en AGL, du plasma $(r=-0,63$ et $-0,78$ respectivement pour 22 données). Les origines de ces modifications peuvent être diverses : variation de la proportion des AG du sang d'origine endogène (réserves corporelles) et exogène (AG absorbés), de l'activité enzymatique de désaturation de la glande mammaire, de la proportion de $\mathrm{C}_{18: 2}$ qui repasse de la glande mammaire dans le sang. 
La concentration des 2 principales fractions lipidiques du plasma, les PL, et les $\mathrm{EC}$, a été liée intra-vaches de façon négative et significative (au moins au niveau Io p. IOO) à la quantité de matières grasses sécrétée par jour et au taux butyreux (tabl. 2). Cela est en opposition avec les résultats de MOORE et al. (I969) et de STEELE et al. (I97I) qui n'ont observé aucune liaison, et de DELAGE et FEHR (I967) qui ont observé une liaison positive. Les essais de ces auteurs différaient cependant sensiblement du nôtre : ils ont été réalisés avec des animaux en milieu de lactation, et avaient pour objet d'étudier l'apport de lipides dans le régime sur la sécrétion des matières grasses du lait. Enfin, nous n'interprétons pas l'évolution du rapport $\mathrm{C}_{18: 2} / \mathrm{C}_{18: s \text {, }}$ qui a d'ailleurs été différente de celle observée par DUNCAN et GARTON (I963) et LEAT et HALI, ( 968$)$.

\section{Les changements métaboliques au début de la lactation et leurs conséquences}

Le début de la lactation s'est accompagné de modifications importantes du métabolisme de la mamelle et du métabolisme général, en particulier en ce qui concerne la sécrétion des matières grasses du lait, le métabolisme lipidique, mais aussi le métabolisme des glucides et de l'azote. Le vêlage ou le début de la sécrétion du lait a en effet provoqué une diminution de la teneur du sang en glucose, ce qu'avaient déjà observé RADLOFF et al. (Ig6o) chez la vache laitière, et de celle du plasma en protéines. Il est possible que les modifications observées soient en partie dues aux changements hormonaux très importants qui ont lieu à la fin de la gestation et au début de la lactation. Mais les liaisons très étroites observées entre la teneur du plasma en glucose et en protéines, et le bilan énergétique (tab1. 2) suggèrent que la cause principale est le déséquilibre entre les apports et les besoins principalement énergétiques et peut-être aussi azotés. Ce déséquilibre peut être très important pour certains métabolites comme le glucose qui est utilisé par la glande mammaire à des fins énergétiques et pour la synthèse du lactose, et comme certains acides aminés indispensables (méthionine, lysine, histidine et acides aminés à chaînes ramifiées) dont le lait est relativement plus riche que les protides du contenu de caillette (CHAMPREDON, I972). Le déficit énergétique permet probablement d'expliquer la baisse de la glycémie qui a entraîné une lipolyse accrue du tissu adipeux, une élévation de la teneur du plasma en AGL et du sang en corps cétoniques.

Ces modifications importantes des teneurs des principaux constituants sanguins au début de la lactation, en particulier, l'augmentation de la teneur en AGL et en corps cétoniques, et la diminution de la teneur en glucose, en protéines (et probablement en certains acides aminés indispensables; Champredon et PION, I972) et des fractions lipidiques autres que les AGL peuvent non seulement être reliées aux modifications de la sécrétion des matières grasses du lait, mais aussi comme il l'a déjà été suggéré (JOURNET et JARRIGE, I970) à l'appétit des animaux. Les teneurs élevées des premiers constituants sont peut-être la cause du faible appétit au début de la lactation, et les teneurs faibles des autres, la cause de l'accroissement de l'appétit. 


\title{
REMERCIEMENTS
}

Nous remercions vivement M. M.-C. Micher, de la Station de Physiopathologie de la Nutrition du C. R. Z. V. de Theix, pour les analyses de glucose et de protéines plasmatiques qu'il a effectuées.

\section{SUMMMARY}

\author{
CHANGES IN CONTENTS OF DIFFERENT BLOOD CONSTITUENTS, \\ PRINCIPALLY THE LIPID FRACTIONS, AT THE END OF GESTATION
} AND THE BEGINNING OF IACTATION. REIATIONS WITH SECRETION OF MILK FAT

In 3 cows in widely different nutritional states we have traced, during the last 3 weeks of gestation and the first 6 weeks of lactation, changes in the concentration of fatty acids of the 4 main classes of plasma lipids and their fatty acid compositions. Changes in blood ketone body content, glucose content and total plasma protein were studied also. We tried to relate this to the amount and composition of the fat secreted in milk and to the nutritional state of the animal.

Concentrations of fatty acids of triglycerides, cholesterol esters and total fatty acids, and of proteins and glucose diminished at the end of gestation, were least at the beginning of lactation and increased after that (fig. I) ; concentrations of free fatty acids and ketone bodies changed in the opposite way.

Free fatty acids in total plasma fatty acids, normally about 5 per cent, reached 20 per cent after calving in the after calving in the cow most seriously underfed (table I). At the same time the proportion of fatty acids in triglycerides was reduced 8-fold.

Of the blood constituents studied, glucose and free fatty acids were best related, within and among cows, to energy balance (table 2).

The amount of milk fat secreted and the fat content of the milk were positively related to the concentration of plasma tree tatty acids (table 2).

The fatty acid composition of the free fatty acids was similar to that of the triglycerides but differed from that of the phospholipids and of the cholesterol esters (table 3 ). In the free fatty acids, the triglycerides and the phospholipids, $C_{18: 0}$ varied in the same direction as the energy balance and $\mathrm{C}_{18: 1}$ varied inversely.

In milk the proportion of long-chain fatty acids (I 8 carbon atoms) was the higher at the start of lactation the greater the energy deficit (or the higher the concentration of free fatty acids) (table 4). The proportion diminished as lactation advanced.

The results are compared with those of other authors and are discussed with particular reference to the synthesis of milk fat.

\section{RÉFÉRENCES BIBLIOGRAPHIQUES}

Annison E. F., Linzell. J. L., Fazakerley S., Nichols B. W., 1967. The oxidation and utilization of palmitate, stearate, oleate and acetate by the mammary gland of the fed goat in relation to their overall metabolism and the role of plasma phospholipids and neutral lipids in milk-fat synthesis. Biochem. J., 102, 637-647.

Annison E. F., Linzell J. L., West C. E., 1968. Mammary and whole animal metabolism of glucose and fatty acids in fasting lactating goats. J. Physiol., 197, 445-459.

Barry J. M., Bartley W., Linzell J. L., Robinson D. S., ig63. The uptake from the blood of triglycerid fatty acids of chylomicra and low-density lipoproteins by the mammary gland to the goat. Biochem. J., 89, 6-I I.

Breirem K., r954. Die Nettoenergie als grundlage der bewertung der futermittel, in : Nehring K., 100 jahre Möckern. Die bewertung der futterstoffe und andere probleme der tiernährung. Berlin, Deutsche Akad. der Landwirtschaftswissenchaften, t. II, 97-I08. 
Carreau J. P., Lapous D., Raulin J., rg69. Artefacts expérimentaux pouvant survenir lors de la chromatographie en couche mince des lipides membranaires. $J$. Chromatog., 42, 422.

Champredon C., Pion R., I972. Évolution de l'aminoacidémie libre des chèvres au début de la lactation. C. R. Soc. Biol., 166, 378 .

Decaen C., Journet M., I967. Évolution, au début de la lactation, de la sécrétion des principaux acides gras du lait et de la concentration en acides gras libres du sang chez la Vache. Ann. Biol anim. Bioch. Biophys., 7, 131-143.

Decaen C., Adda J., I970. Évolution de la sécrétion des acides gras des matières grasses du lait au cours de la lactation de la Vache. Ann. Biol. anim. Bioch. Biophys., 10, 659-677.

DELAGE J., FEHR P.-M., rg67. Influence des lipides alimentaires sur la sécrétion des acides gras par la mamelle de Chèvre. II. Influence de l'addition d'acides gras en $\mathrm{C}_{18}$ à des régimes pauvres en lipides, sur la composition lipidique du plasma sanguin et du lait. Ann. Biol. anim. Bioch. Biophys., 7, 445-457.

Demarquilly C., I97o. Tableaux de la valeur alimentaire des fourrages. (Édité par le S. E. I. de 1'I. N.R. A., route de Saint-Cyr, 78 Versailles).

Dole V. P., Meinertz H., I960. Determination of long chain fatty acids in plasma and tissues. J. Biol. Chem., 235, 2595-2599.

Duncan W. R. H., Garton G. A., I963. Blood lipids. 3. Plasma lipids of the cow during pregnancy and lactation. Biochem. $J ., 88,414-419$.

Evans L., Patton S., Mc Carthy R. D., r96I. Fatty acid composition of the lipid fractions from bovine serum lipoproteins. J. Dairy Sci., 44, 475-482.

Fisher L. J., ERfLE J. D., SAUER F. D., I97I. Inducement of ketotic symptoms in lactating cows by reducing their plane of nutrition. Can.J. Anim. Sci., 51, 153-160.

FlanzY J., r969. Communication personnelle.

Folch J., Lees M., Sloane-Stanley G. H., 1957. A simple method for the isolation and purification of total lipids from animal tissues. J. Biol. Chem., 226, 497-509.

GHADAKI M. B., I968. Influence de quelques facteurs alimentaires sur la sécrétion des principaux acides gras $d u$ lait de vache. Thèse de Docteur-Ingénieur. Clermont-Ferrand.

Hartmann P. E., Lascelles A. K., 1964. The uptake of plasma lipid and some non lipid constituents by the mammary gland of the cow. Aust. J. Biol. Sci., 17, 935-944.

Hartmann P. E., Lascelles A. K., 1965. Variation in the concentration of lipids and some other constituents in the blood plasma of cows at various stages of lactation. A ust. J. Biol. Sci., 18, II4-I23.

Hartmann P. E., Lascelles A. K., I 665 . The effect of starvation on the uptake of the precursors of milk fat by the bovine mammary gland. A ust. J. Biol. Sci., 18, ro25-1034.

HrLditch T. P., Williams P. N., 1964. The chemical constitution of natural fats. $4^{e}$ edition. Chapman et Hall-Londres.

Journet M., JARRIGe R., I970. Facteurs physiologiques de la quantité d'aliment ingérée par les Ruminants. $X X I^{\mathrm{e}}$ Congrès anmuel de la Fédération Européenne de Zootechnie. Budapest.

KRONFELD D. S., I 965 . Plasma non-esterified fatty acid concentrations in the dairy cow : responses to nutritional and hormonal stimuli, and significance in ketosis. Vet. Rec., 77, I-6.

LEAT W. M. F., rg66. Fatty acid composition of the plasma lipids of newborn and maternal ruminants. Biochem. J., 98, 598-603.

Leat W. M. F., HaLl J. G., r968. Lipid composition of lymph and blood plasma of the cow. J. agric. Sci., I8g-r94.

Lennon Jr H. D., Mixner J. P., 1957. Some sources of variation in total plasma cholesterol levels in dairy cattle. J. Dairy Sci., 40, I424-1429.

LEROY A-M., r949. Ve Congrès Intern. de Zootechnie, Paris, 1.

Mahadevan V., Lundberg W. O., r962. Preparation of cholesterol esters of long chain fatty acids and characterization of cholesteryl arachidonate. J. Lipid Res., 3, ro6-1 ro.

Michel M.-C., I97I. Analyse quantitative de quelques substances azotees et glucidiques en milieu biologique. Essai de rationalisation. Thèse Clermont-Ferrand, p. 63.

Moore J. H., Steele W., Noble R. C., I969. The relationships between dietary fatty acids, plasma lipid composition and milk fat secretion in the cow. J. Dairy Res., 36, 383-392.

MoRRIs, r967. Communication personnelle.

Procos J., rg6r. Modification of the spectrophotometric determination of ketone bodies on blood enabling the total recovery of $\beta$-hydroxybutyric acid. Clin. Chem., 7, 97-106.

Radioff H. D., Schultz L. H., Hoekstra W. G., I966. Relationship of plasma free fatty acids to other blood components in Ruminants under various physiological conditions. J. Dairy Sci., 49, 179-182.

Rémond B., Journet M., I97o. Influence de l'état nutritionnel des vaches laitières au début de la lactation sur la mobilisation des réserves lipidiques et la sécrétion des matières grasses du lait. Congrès International de Laiterie, B 6 p. 6r9. Sydney.

Snedecor G. W., Cochran W. G., 1957. Statistical methods. 6e edition Iowa State University Press. Ames. Iowa. 
Stener W. NoBLE R. C, MooRE J, H, I971, The relationship between plasma lipid composition and milk fat secretion in cows given diets containing soybean oil. J. Dairy Res, 88, $57-64$.

Tove S. B., 1965. In DovgreRTY R. W, Physiology of digestion in the numinant, $399 \cdot 4$ ro, Butterworths, Washington.

VARMAN P, N SCHULTZ L, H, I968. Blood lpids of cows at different stages of lactation. J. Daivy Sci, 51, 197I-1974. 